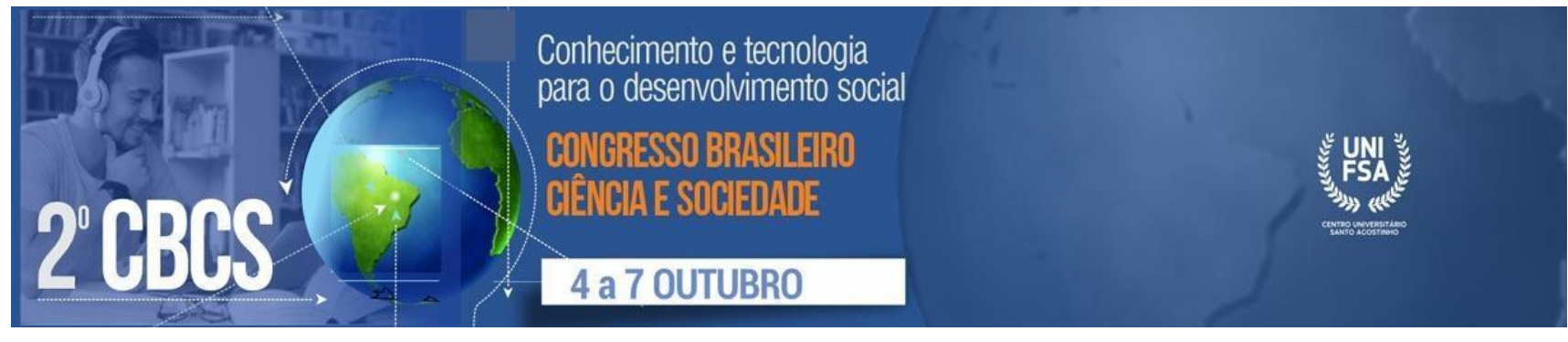

\title{
ACOMPANHAMENTO FARMACOTERAPÊUTICO A USUÁRIOS DE HIDROXICLOROQUINA PARA O TRATAMENTO DO COVID-19
}

\author{
Juliane da Silva Diniz- (Acadêmica do curso de farmácia, UNIFSA); \\ Khryscia Teresa Lima da Cunha- (Acadêmica do curso de farmácia, UNIFSA); \\ Willian Assunção Costa Sá- (Acadêmico do curso de farmácia, UNIFSA); \\ Manoel Pinheiro Lúcio Neto - (Professor, doutor do curso de farmácia, UNIFSA);
}

\section{Resumo}

Objetivos: Descrever e Analisar a dispensação de hidroxicloroquina para o tratamento da COVID-19 em um hospital terciário do nordeste brasileiro. Métodos: Pesquisa descritiva com abordagem quantitativa e qualitativa. A coleta de dados ocorreu com análise de prontuários. Resultados: $\mathrm{O}$ uso da hidroxicloroquina ocorreu em aproximadamente $69 \%$ dos pacientes e seu uso em associação com azitromicina ou outros antibióticos está relacionado com a presença ou não sintomas relacionados à infecção por covid-19. CONSIDERAÇÕES FINAIS: O uso da hidroxicloroquina bem como sua associação com outras medicações ainda necessita de mais pesquisas para justificar e consolidar seu uso no tratamento da covid-19, o seu uso indicado em alguns países impulsionou a aplicação da mesma de forma experimental durante a pandemia e traz discursões clínicas e políticas, neste presente estudo pode-se observar seu uso e algumas das implicações na prática clínica.

Palavras-chave: Hidroxicloroquina. Covid-19. Farmacêutico.

\section{Introdução}

Em 31 de dezembro de 2019, a Organização Mundial da Saúde (OMS) foi notificada sobre uma nova doença viral respiratória identificada em Wuhan, na China. Em 12 de março de 2020, a OMS declarou que o mundo estava enfrentando uma nova pandemia. O sequenciamento genético sugeriu ser um betacoronavírus ligado ao vírus da SARS (SARSCoV-2) 1, que ocasiona a doença por coronavírus 2019 (COVID-19). Esse vírus liga-se à enzima ECA2 2, presente nas células humanas e apresenta grande poder de disseminação entre humanos. (WIERSINGA WJ; RHODES A; CHENG AC et al., 2020). 
Dados preliminares de estudos in vitro identificaram atividades antivirais dos fármacos cloroquina e hidroxicloroquina, associados a antibióticos macrolídeos como azitromicina, e estes foram recomendados em alguns países como terapia medicamentosa contra o SARS-CoV-2. Apesar de esses fármacos serem indicados para outras doenças, o uso nesta pandemia é experimental, e mesmo o uso compassivo pode representar riscos à saúde devido ao potencial de causar reações adversas, principalmente o risco de cardiotoxicidade. (GÉRARD et al., 2020; SAMPAIO et al., 2020; KALIL et al., 2020).

Recentemente, resultados mostraram atividade da cloroquina (CQ) contra o SARSCoV-19 in vitro, além disso, trouxe a recomendação de alguns especialistas e pesquisadores sobre a eficácia dessa droga em pacientes com a COVID-19. Por isso, a Administração de Alimentos e Medicamentos dos Estados Unidos (FDA) tem trabalhado na investigação da CQ para uso na COVID-19. Como um derivado da CQ, a hidroxicloroquina (HCQ) tem efeitos terapêuticos similares e menos efeitos adversos. Baseado em suas características de regulação imunológica, atividade antitrombótica e melhora inflamatória, HCQ tem sido usada, rotineiramente, no tratamento clínico do Lúpus Eritematoso Sistêmico (LES). Entretanto, a eficácia da HCQ na COVID-19 permanece desconhecida (J. GAO et al., 2020)

A hidroxicloroquina e cloroquina foram os dois únicos medicamentos que estiveram estatisticamente associados à presença de reações adversas medicamentosas (RAM) graves. Pacientes que usaram a hidroxicloroquina registraram quase 2 vezes mais chances de apresentar RAM grave do que aqueles que não usaram este medicamento e, de uma forma mais agravada, os que fizeram o uso da cloroquina registraram quase 6 vezes mais chances de apresentar RAM grave, comparados aos que não usaram este medicamento. (STALLIN et al., 2020; MELO et al., 2020).

Diante disso os farmacêuticos entraram numa verdadeira corrida contra o tempo na busca pelo tratamento e cura do Coronavírus, desde então não parou mais protagonizando iniciativas essenciais no combate ao tratamento, por meio de parcerias com governos e organizações internacionais, doações de medicamentos e outras ações focadas no paciente na contenção do vírus. Além de pesquisas inovadoras em medicamentos, vacinas e diagnóstico ainda não há cura, para chegar a um tratamento de fato é necessárias inúmeras pesquisas clinicas para consolidar de fato a cura dessa doença. Além disso, os profissionais farmacêuticos torna-se um elo importante de acesso aos cuidados em saúde, tendo em vista que é o profissional responsável pelo processo de produção e dispensação de medicamento. 
Em função de suas competências é considerado um profissional estratégico tanto no combate quanto no tratamento do paciente portador do COVID 19 (UNG, 2020).

Portanto, esta pesquisa tem como objetivo analisar a dispensação de hidroxicloroquina para o tratamento da COVID-19 em um hospital terciário do nordeste brasileiro.

\section{Metodologia}

A pesquisa é do tipo descritivo, quantitativo e qualitativo, realizado em um Hospital em Teresina PI, local que possui uma alta referência para as regiões do Nordeste e que presta serviços ao Sistema Único de Saúde. Esta coleta de dados ocorreu no período de março a junho de 2021, após a autorização da instituição. Foi uma pesquisa realizada com base de dados no programa estatístico SPSS 20.0. E para a realização desta pesquisa foram incluídos os profissionais farmacêuticos da farmácia hospitalar que atendem pacientes com suspeitas de COVID-19, e excluindo assim os demais funcionários.

Foram utilizados os prontuários dos pacientes para coleta de dados, esta coleta ocorreu com auxílio dos farmacêuticos do hospital supracitado, e direcionado à prescrição de medicamento utilizado para o tratamento da COVID-19, a coleta também levou em consideração a evolução do quadro clínico dos pacientes para compor os resultados desta pesquisa, sendo dividida em dois momentos:

\begin{tabular}{|lll|lll|}
\hline \multicolumn{2}{|l|}{ MOMENTO O1 } & & MOMENTO 02 & & \\
\hline PRESCRIÇÃO & DE & HIDROXICLOROQUINA & E & EVOLUÇÃO CLÍNICA DO PACIENTE \\
ASSOCIAÇÃO & DE & HIDROXICLOQUINA COM & (Sintomáticos e Assintomáticos). & \\
OUTROS MEDICAMENTOS. & & & & \\
\end{tabular}

A análise dos dados ocorreu com o processamento dos dados no programa estatístico SPSS 20.0 para a confecção de gráficos e porcentagens para posterior discussão com a literatura científica referente ao tema estudado. Estes dados não podem ser divulgados ou anexados nesta pesquisa por motivos éticos e normas do hospital.

A principal limitação do presente estudo encontra-se na amostragem temporal em que ocorreu a coleta de dados.

\section{Resultados e Discussão}


Em um hospital da cidade de Teresina-PI, realizamos uma pesquisa fazendo o levantamento de dados de resultados dos pacientes que fizeram tratamento com a hidroxicloroquina para o tratamento do COVID-19, onde foi feito um levantamento do perfil do paciente, quanto aos sintomas apresentados nos pacientes assistidos e a dispensação de medicamentos, assim podendo observar nos gráficos abaixo.

Essa pesquisa tem como principal objetivo observar o medicamento estudado e consumido pelos pacientes e de acordo com isso tivemos resultados parcial pelos pacientes que fizeram o consumo deste medicamento.

Apresenta-se uma visão panorâmica do conhecimento atual relacionado ao tratamento e alternativas terapêuticas para COVID-19, desde medicamentos que têm sido usados frequentemente em outras doenças com comprovada atividade antiviral, até medicamentos que geralmente têm sido usados como antibacterianos, mas que, na situação atual, são mais uma vez evidente por sua possível e potencial eficácia no tratamento da COVID-19.

Gráfico 1 - Atendimentos de pacientes por dia.

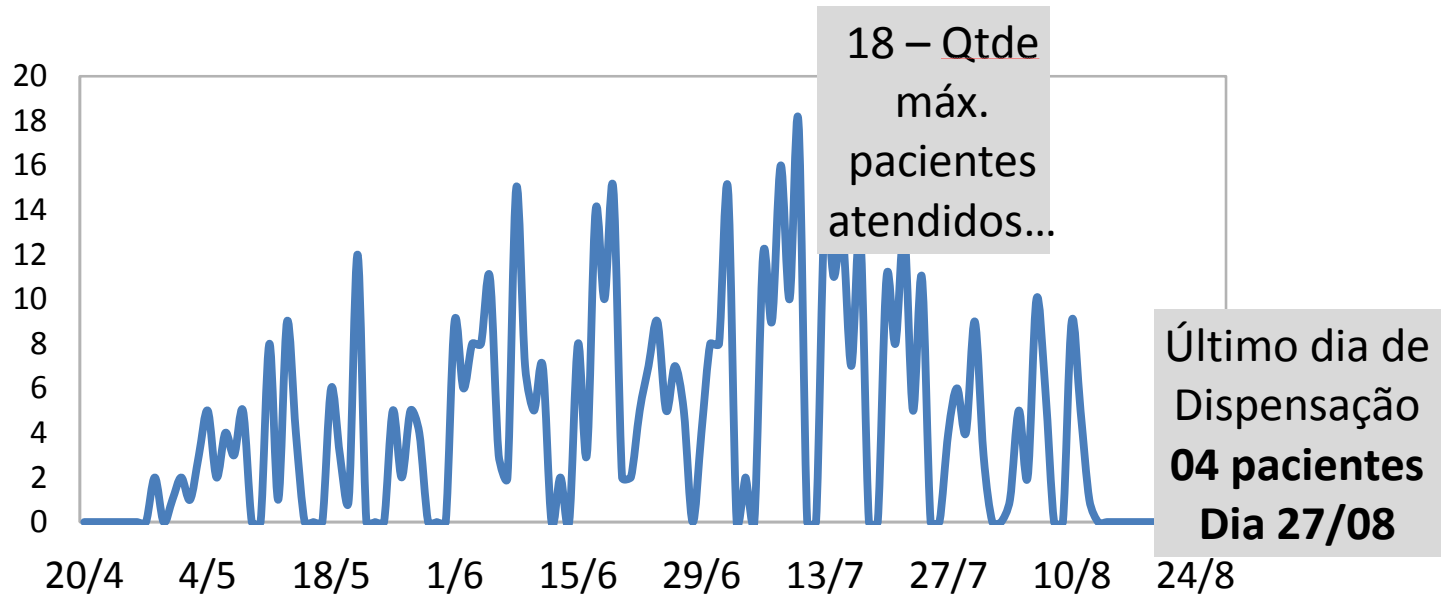

Fonte: dados da pesquisa realizada (2021) - Diniz; J.S; Cunha; K.T.L.

De acordo com a pesquisa realizada o atendimento de cada paciente realizado por dia, sendo assim, pode-se observar como seria o uso da hidroxicloroquina nesses pacientes. O gráfico 1 ele apresenta em média o atendimento de cada paciente por dia a partir de uma quantidade máxima vista em pacientes atendidos em um único dia. E a dispensação da hidroxicloroquina para o tratamento desse paciente. 
A ciência diz que a COVID-19 é uma doença muito recente, e que estudos clínicos randomizados levam algum tempo para ser realizados com um número significante de indivíduos. Esse aspecto gera um impasse na adoção de novos tratamentos para COVID-19. (COLSON et al., 2020).

Podemos observar no gráfico 2 que os maiores números de pessoas atendidas foram do sexo feminino sendo $59 \%$ e de menor número $41 \%$ do sexo masculino, sendo eles assintomáticos com menor porcentagem de $22 \%$ e com sintomas de maior porcentagem de $78 \%$.

De acordo com os atendimentos realizados em cada mês como vimos na pesquisa, foi diagnosticado como uso para tratamento da COVID-19 a hidroxicloroquina para o total de 554 pessoas entre os meses de abril a agosto, sendo eles do sexo feminino ou masculino, sendo assintomáticos ou com sintomas (Gráfico 3).

\section{Gráfico 2 - Perfil dos pacientes}
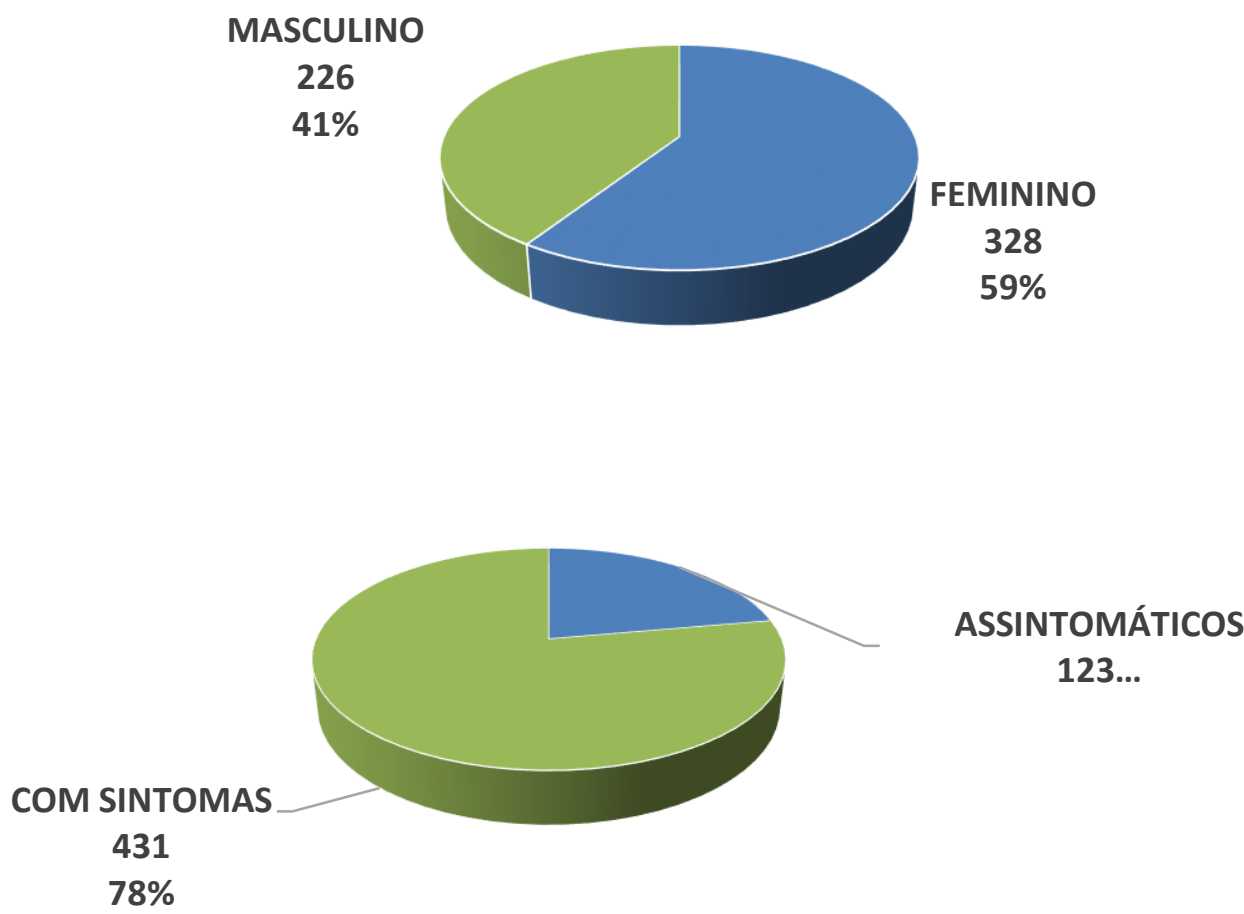

Fonte: dados da pesquisa realizada (2021) - Diniz; J.S; Cunha; K.T.L. 
Gráfico 3 - Atendimentos de pacientes por mês

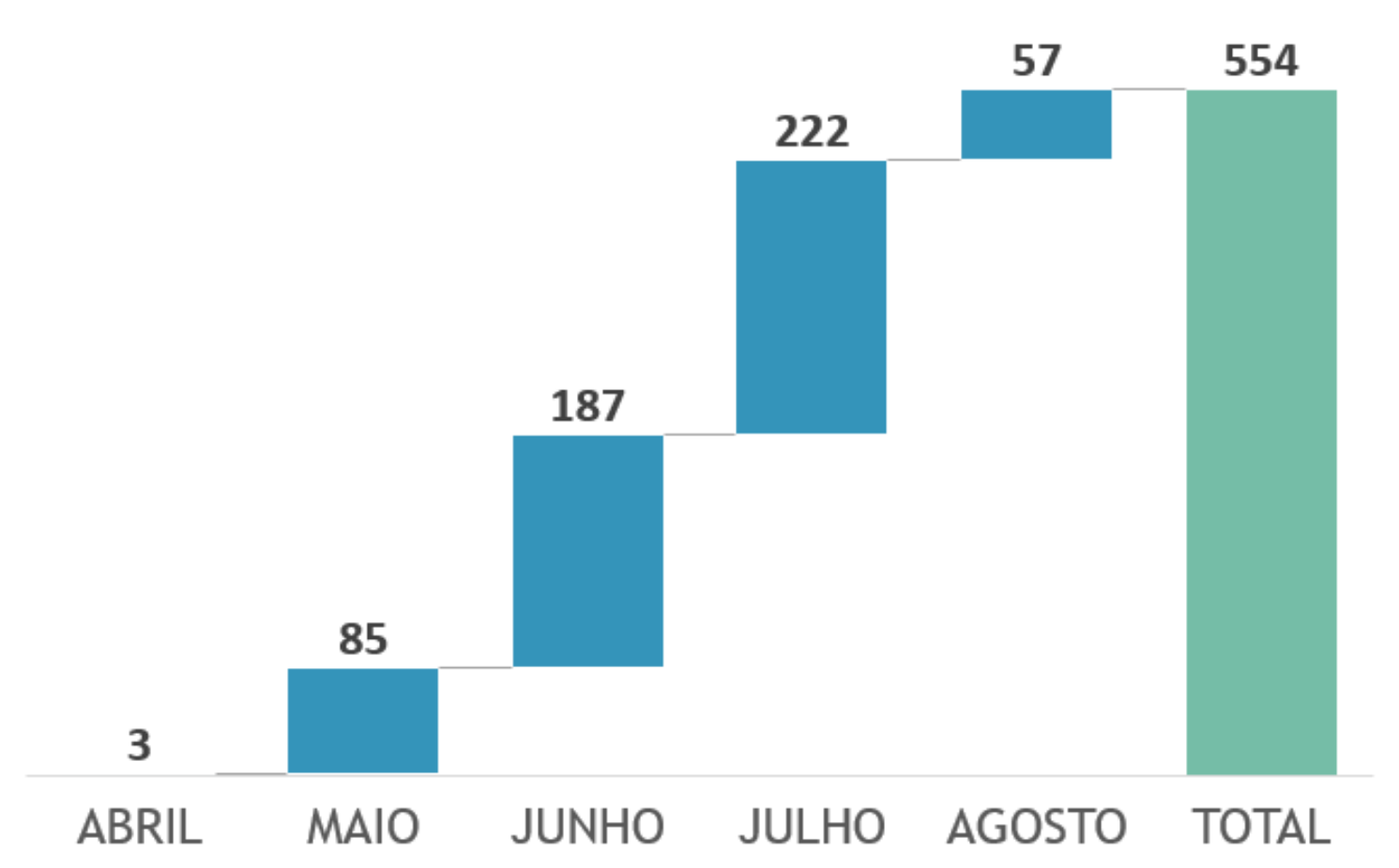

Fonte: dados da pesquisa realizada (2021) - Diniz; J.S; Cunha; K.T.L.

Todavia, foi demonstrado que a hidroxicloroquina tem uma potencial resposta antiviral in vitro contra o SARS-CoV2 causador da COVID-19 e um aparente bom resultado na clínica. Portanto, é urgente avaliar melhor a eficácia desse possível tratamento de baixo custo para curar pacientes em um estágio inicial, antes das complicações respiratórias graves irreversíveis (SCHEREZENMEIER et al., 2020).

O farmacêutico encontra vários desafios ao enfrentamento do Covid-19, sendo um trabalho intenso, onde o farmacêutico faz a separação de medicamentos faz todo o papel de distribuição para os setores sendo responsável fazendo todo acompanhamento farmacoterapêutico (SILVA, 2020)

Os profissionais farmacêuticos são importantes para a saúde durante a pandemia do novo coronavírus servindo para seus pacientes como profissionais na linha de frente. Se tornando mais fácil o acesso com os medicamentos e assim evitando os efeitos adversos. Portanto, observamos que cada paciente que é examinado pelo médico ele sempre apresenta mais de um sintoma que faz parte dos sintomas apresentados por uma pessoa que possui COVID-19. De acordo com os pacientes notificados pelo Sistema de Notificação 
da ANVISA (NOTEVISA) 47 desses pacientes eles apresentam sintomas de arritmia e 217 desses pacientes apresentam diarreia. (Gráfico 4)

Gráfico 4 - Quanto aos sintomas apresentados nos pacientes assistidos.

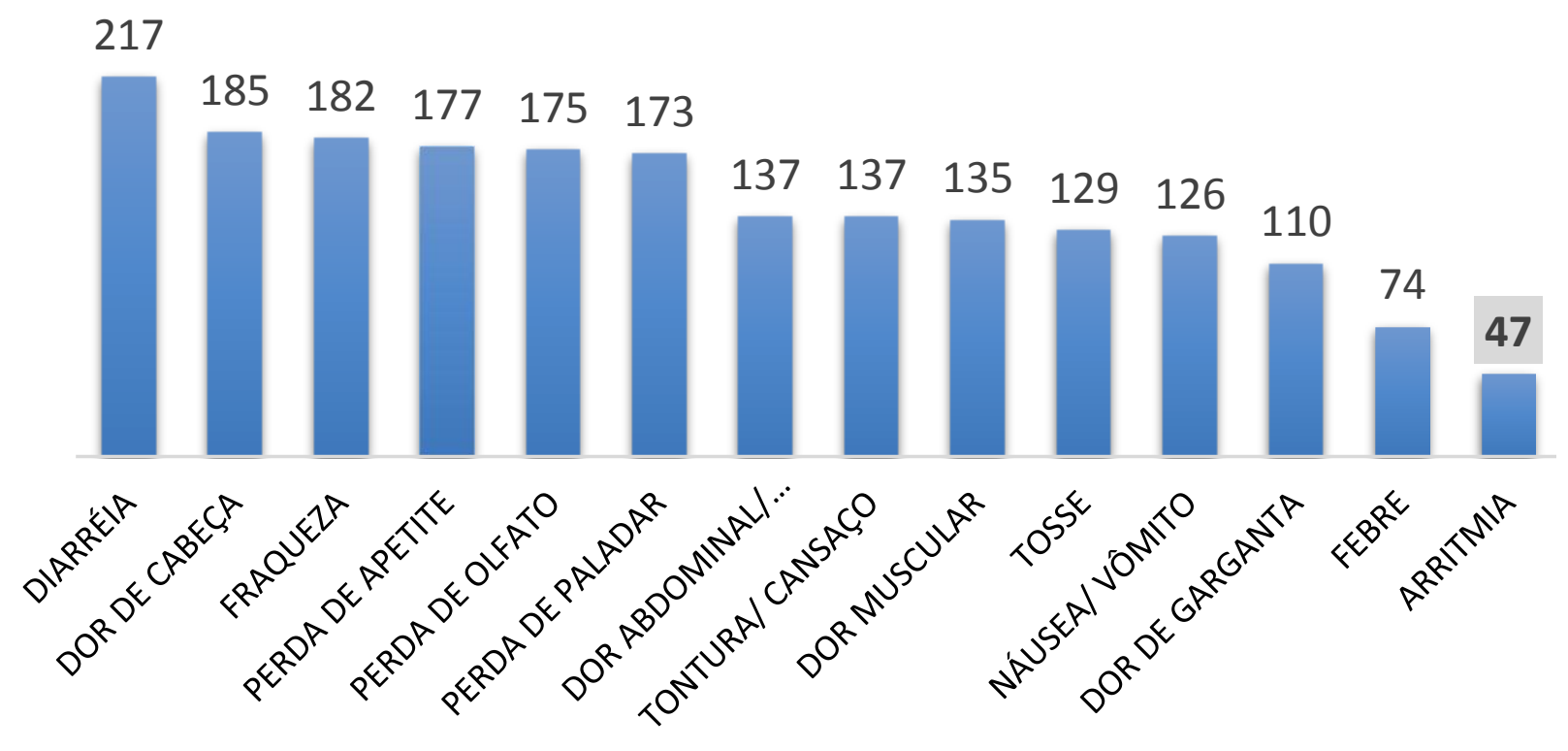

Fonte: dados da pesquisa realizada (2021) - Diniz; J.S; Cunha; K.T.L.

Como o paciente ele apresenta mais de um sintoma os medicamentos a serem dispensados foram sempre a hidroxicloroquina + azitromicina ou azitromicina entre outros medicamentos que possam ajudar no tratamento da COVID-19, de acordo com os sintomas de cada paciente.

A ocorrência de sintomas como descritas no gráfico, estão diretamente relacionados com a associação ou não da hidroxicloroquina com outros medicamentos como a azitromicina. Para (MANUEL, 2020) esta associação é importante para conter o progresso da infecção causada pelo novo coronavírus.

Na dispensação realizada pelo farmacêutico, é de extrema importância observar que os medicamentos que possuem um melhor resultado no tratamento de 294 pacientes é a hidroxicloroquina + azitromicina. Tendo então um resultado positivo diante das outras medicações.

Nesse sentido, Zhou e colaboradores (2020), propõem que a hidroxicloroquina, que apresenta um efeito antiviral muito semelhante ao da cloroquina, poderia servir como uma melhor abordagem terapêutica. A hidroxicloroquina provavelmente atenua a progressão 
grave da COVID-19, inibindo a tempestade de citocinas ao suprimir a ativação das células T. Além disso, tem um perfil clínico mais seguro e é adequada para gestantes.

Gautret e colaboradores (2020), publicaram os resultados de um ensaio clínico aberto, não randomizado, no qual avaliaram o papel da hidroxicloroquina nas cargas virais respiratórias. Os pacientes franceses com COVID-19 foram incluídos no estudo desde o início até 16 de março. Diariamente, eles receberam $600 \mathrm{mg}$ de hidroxicloroquina e suas cargas virais também foram testadas em swabs nasofaríngeos. De acordo com o quadro clínico, azitromicina foi adicionada ao tratamento. Pacientes não tratados de outro centro e casos que não aceitaram a aplicação do protocolo foram incluídos como controles negativos.

Dessa forma, o profissional farmacêutico tem a função de possuir serviços da assistência farmacêutica se baseando em indícios para compromete-se ao sistema de abastecimento de medicamentos sendo assim para possuir controle de medicamentos para um bom atendimento ao paciente.

Gráfico 5 - Dispensação de Medicamentos

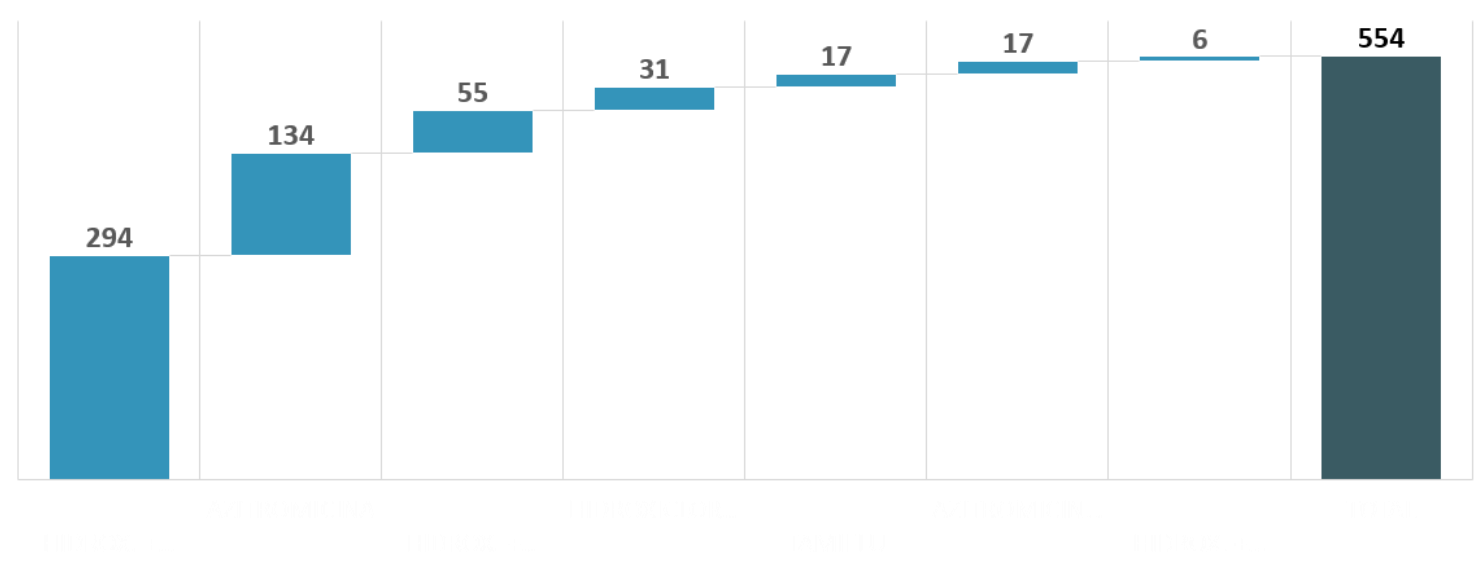

\begin{tabular}{|l|l|l|l|l|l|l|l|}
\hline $\begin{array}{l}\text { HIDROX. } \\
+ \\
\text { AZITRO- } \\
\text { MICINA }\end{array}$ & $\begin{array}{l}\text { AZITRO- } \\
\text { MICINA }\end{array}$ & $\begin{array}{l}\text { HIDROXI.+ } \\
\text { AZITROMI- } \\
\text { CINA+TAMI- } \\
\text { FLU. }\end{array}$ & $\begin{array}{l}\text { HIDROXI- } \\
\text { CLOROQUINA }\end{array}$ & TAMIFLU & $\begin{array}{l}\text { AZITROMI. } \\
+ \\
\text { TAMIFLU }\end{array}$ & $\begin{array}{l}\text { HRIDROXI. } \\
+ \\
\text { TAMIFLU }\end{array}$ & TOTAL \\
\end{tabular}

Fonte: dados da pesquisa realizada em (2021) - Diniz; J.S; Cunha; K.T.L.

* Um paciente geralmente apresenta mais de um sintoma

\section{Considerações Finais}

A utilização da hidroxicloroquina associado com antibióticos foi recomendada para o tratamento do SARS-CoV2 em alguns países e embora a utilização destes medicamentos seja 
indicada no tratamento de outras patologias, estes foram utilizados no período de pandemia de forma experimental no combate à covid-19. De acordo com esta utilização clínica, o presente estudo buscou entender o acompanhamento farmacêutico nos pacientes em uso das medicações supracitadas com dados coletados em prontuários e análise qualitativa e quantitativa dos mesmos. Os dados encontrados demonstraram a utilização mensal de hidroxicloroquina por aproximadamente $69 \%$ dos pacientes atendidos mensalmente na unidade. Nas dispensações realizadas pelo farmacêutico (a) notou-se uma melhor resposta quando a hidroxicloroquina foi associado com a Azitromicina, exercendo a função de controle de medicamentos com melhores resultados, sendo assim, prestando assistência farmacêutica no combata à covid-19 e contribuindo para a construção de conhecimento científico em saúde para auxiliar de forma ativa na conduta terapêutica adotada pelas equipes de saúde.

\section{REFERÊNCIAS}

COLSON, Philippe et al. Chloroquine and hydroxychloroquine as available weapons to fight COVID-19. Int J Antimicrob Agents, v. 105932, n. 10.1016, 2020.

Gérard A, Romani S, Fresse A, Viard D, Paras - sol N, Granvuillemin A, et al. "Off-label" use of hydroxychloroquine, azithromycin, lopina - vir-ritonavir and chloroquine in COVID-19: a survey of cardiac adverse drug reactions by the French Network of Pharmacovigilance Cen ters. Therapie; v. 75 p. 371-379,2020.

GAUTRET, Philippe et al. Hidroxicloroquina e azitromicina como tratamento de COVID-19: resultados de um ensaio clínico não randomizado de rótulo aberto. Revista Internacional de Agentes Antimicrobianos, v. 105949, 2020.

Indicators related to the rational use of medicines and its associated factors; Rev Saude Publica. 2017; 51(Suppl 2): 23s. Published online 2017 Sep 22.

International Pharmaceutical Federation (FIP). Coronavirus SARS-CoV-2/COVID-19 pandemic: Information and interim guidelines for pharmacists and the pharmacy workforce (Atualizado 19/03/2020). 2020;1-48.

J. Gao, Z. Tian, X. Yang. Breakthrough: chloroquine phosphate has shown apparent efficacy in treatment of COVID-19 associated pneumonia in clinical studies. Biosci Trends (2020 Feb 19), 10.5582/bst.2020.01047. DOI: 10.5582/bst.2020.01047. 
Kalil AC. Treating COVID-19-off-label drug use, compassionate use, and randomized clinical trials during pandemics. JAMA 2020; 323:1897-8.

Melo JRR, Duarte EC, Ferreira KDA, Gonçalves YS, Moraes MVD, Arrais PSDR. Assessment of knowledge, attitude and practice of pharmacovigilance among healthcare professionals in Brazil. J Young Pharm 2020; 12:255-60.

Satlin MJ, Goyal P, Magleby R, Maldarelli GA, Pham K, Kondo M, et al. Safety, tolerability, and clinical outcomes of hydroxychloroquine for hospitalized patients with coronavirus 2019 disease. PLoS One 2020; 15:e0236778.

Schrezenmeier E, Dörner T. Mechanisms of action of hydroxychloroquine and chloroquine: implications for rheumatology. Nat Ver Rheumatol [Internet]. 2020 Mar [acesso 2020 Abr 20]; 16(3): 155-166.

SILVA, B. C.; OLIVEIRA, J. V. A importância da atuação permanente do farmacêutico na equipe multidisciplinar da UTI em benefício da saúde do paciente e redução de custos para um hospital no município de Imperatriz-MA. Monografia de conclusão do curso de farmácia (Graduação em Farmácia), Faculdade Imperatriz, 2016.

The good pharmacy practice on Einstein Program at Paraisópolis Community; Einstein (Sao Paulo). 2016 Jul-Sep; 14(3): 415-419.Wiersinga WJ, Rhodes A, Cheng AC, Peacock SJ, Prescott HC. Pathophysiology, transmis - sion, diagnosis, and treatment of coronavirus disease 2019 (COVID-19): a review. JAMA 2020; 324:782-93

Wiedenmayer K, Summers RS, Mackie CA, Gous AGS, Everard M.

Developingpharmacypractice: a focusonpatientcare: handbook 2016. Geneva: World Health OBrganization: InternationalPharmaceuticalFederation.

World Health Organization. Coronavirus disease (COVID-19). Weekly Epidemiological Update - 6 September 2020. Acesso em 11 de setembro 2020.

UNG, C. O. L. Community pharmacist in public health emergencies: Quick to action Against the coronavirus 2019-nCoV outbreak. Research in social and Administrative Phamarcy. v. 16, n. 4, p.583-586, 2020.

Zhou D, Dai SM, Tong Q. COVID-19: uma recomendação para examinar o efeito da hidroxicloroquina na prevenção da infecção e progressão. J Antimicrob Chemother. 2020. 